\title{
Imbalance of neurotrophin receptor isoforms TrkB-FL/TrkB-T1 induces neuronal death in excitotoxicity
}

\author{
ÓG Vidaurre ${ }^{1,7}, \mathrm{~S}$ Gascón ${ }^{1,3,7}, \mathrm{R}$ Deogracias ${ }^{1,4}$, M Sobrado ${ }^{1,5}$, E Cuadrado ${ }^{2,6}$, J Montaner $^{2}$, Á Rodríguez-Peñaa and M Díaz-Guerra ${ }^{\star 1}$
}

A better understanding of the mechanisms underlying neuronal death in cerebral ischemia is required for the development of stroke therapies. Here we analyze the contribution of the tropomyosin-related kinase B (TrkB) neurotrophin receptor to excitotoxicity, a primary pathological mechanism in ischemia, which is induced by overstimulation of glutamate receptors of the $\mathrm{N}$-methyl-D-aspartate type. We demonstrate a significant modification of TrkB expression that is strongly associated with neurodegeneration in models of ischemia and in vitro excitotoxicity. Two mechanisms cooperate for TrkB dysregulation: (1) calpain-processing of full-length TrkB (TrkB-FL), high-affinity receptor for brain-derived neurotrophic factor, which produces a truncated protein lacking the tyrosine-kinase domain and strikingly similar to the inactive TrkB-T1 isoform and (2) reverse regulation of the mRNA of these isoforms. Collectively, excitotoxicity results in a decrease of TrkB-FL, the production of truncated TrkB-FL and the upregulation of TrkB-T1. A similar neuro-specific increase of the TrkB-T1 isoform is also observed in stroke patients. A lentivirus designed for both neuro-specific TrkB-T1 interference and increased TrkB-FL expression allows recovery of the TrkB-FL/TrkB-T1 balance and protects neurons from excitotoxic death. These data implicate a combination of TrkB-FL downregulation and TrkB-T1 upregulation as significant causes of neuronal death in excitotoxicity, and reveal novel targets for the design of stroke therapies.

Cell Death and Disease (2012) 3, e256; doi:10.1038/cddis.2011.143; published online 19 January 2012

Subject Category: Neuroscience

Stroke is a leading cause of death, dementia, and adult disability. The available therapies are insufficient to restrict brain damage; therefore, we need to understand the mechanisms underlying neuronal death in those regions surrounding the infarct. A primary pathological mechanism in ischemia is excitotoxicity, a form of neuronal death induced by overactivation of the N-methyl-D-aspartate (NMDA)-type of glutamate receptors (NMDARs), also associated with other acute disorders or neurodegenerative diseases. ${ }^{1}$ Nonetheless, efforts to inhibit NMDARs have generally failed, mainly due to the critical role these receptors have in neuronal survival, ${ }^{2,3}$ synaptic plasticity, learning, and memory. ${ }^{4}$ Prosurvival signaling initiated by synaptic NMDARs includes induction of antioxidant defenses, ${ }^{5}$ activation of extracellular signal-regulated kinase, ${ }^{6}$ and phosphorylation of the cAMP response element-binding protein (CREB), which results in the expression of brain-derived neurotrophic factor (BDNF) $)^{7,8}$ and its receptor, tropomyosin-related kinase $B$ (TrkB).,10 Pathological activation of NMDARs opposes these effects by coupling $\mathrm{Ca}^{2+}$-influx to mitochondrial dysfunction and neuronal death pathways. $^{2,11}$ Additionally, NMDAR overactivation also disrupts critical survival pathways by uncoupling synaptic NMDARs from cytoskeletal proteins and pro-survival signaling. Transcriptional downregulation of the NMDAR subunit, GluN1, ${ }^{12}$ and calpain-processing of the GluN2 subunits ${ }^{13,14}$ and NMDAR-interacting proteins ${ }^{13,15}$ in excitotoxicity and ischemia have been demonstrated. Calpain is an effector of $\mathrm{Ca}^{2+}$-overload central to ischemia acting as a modulatory protease that transforms substrate stability, location, or activity. ${ }^{16}$

Processes downstream of the NMDAR overactivation, which are aberrant in stroke, might constitute novel therapeutic targets for reduction of neuronal death. We hypothesized that neurotrophin receptors, which are associated with NMDARs, ${ }^{17,18}$ are modified by ischemia as other NMDARrelated proteins. Although neurotrophins have been proposed as therapeutic agents for stroke and excitotoxicity-associated diseases, ${ }^{19}$ the effect of ischemia on neurotrophin signaling is

\footnotetext{
${ }^{1}$ Instituto de Investigaciones Biomédicas 'Alberto Sols', CSIC-UAM, Madrid, Spain and ${ }^{2}$ Neurovascular Research Laboratory, Hospital Vall d'Hebron, Passeig Vall d'Hebron, Barcelona, Spain

${ }^{*}$ Corresponding author: M Díaz-Guerra, Instituto de Investigaciones Biomédicas 'Alberto Sols', CSIC-UAM, Arturo Duperier 4, 28029-Madrid, Spain.

Tel: + 341 5854443; Fax: 341 5854401; E-mail: mdiazguerra@iib.uam.es

${ }^{3}$ Current address: Helmholtz Zentrum München, German Research Center for Environmental Health (GmbH), Institute of Stem Cell Research, Ingolstädter Landstrasse 1, D-85764 Neuherberg/Munich, Germany.

${ }^{4}$ Current address: Biozentrum, University of Basel. Klingelbergstrasse 50/70, $\mathrm{CH}-4056$ Basel, Switzerland.

${ }^{5}$ Current address: Unidad de Ictus, Servicio de Neurología, Hospital La Princesa, Madrid, Spain.

${ }^{6}$ Current address: Netherlands Institute for Neuroscience. Meibergdreef 47, 1105 BA Amsterdam, The Netherlands.

${ }^{7}$ These authors contributed equally to this work.

Keywords: calpain; excitotoxicity; ischemia; neurotrophins; NMDA; TrkB

Abbreviations: ARMS, ankyrin repeat-rich membrane spanning; BDNF, brain-derived neurotrophic factor; BDPs, breakdown products; DIV, Days in vitro; Kidins220, Kinase D-interacting substrate of $220 \mathrm{kDa}$; MCAO, middle cerebral artery occlusion; NMDA, N-Methyl-D-aspartate; NMDAR, NMDA receptor; TrkB, tropomyosin-related kinase B

Received 25.7.11; revised 15.11.11; accepted 14.12.11; Edited by A Verkhratsky
} 
largely unknown. These molecules are fundamental for the development of adult nervous system. Among them, BDNF has a major role through binding to its high-affinity tyrosinekinase receptor, TrkB, and initation of signaling cascades that regulate gene expression, neuronal survival, neurotransmitter release, and synaptic transmission. ${ }^{20}$ In addition to the full-length isoform (TrkB-FL) encoding the active receptor, alternative splicing yields receptors that lack the kinase domain, TrkB-T1, TrkB-T2, and TrkB-T-Shc, ${ }^{21}$ suggested as involved in TrkB-FL modulation. ${ }^{22}$ Particularly, TrkB-T1 opposes TrkB-FL function ${ }^{23,24}$ via competition for BDNF binding $^{21}$ or the formation of inactive heterodimers. ${ }^{25}$ Additionally, TrkB-FL-independent signaling has been also suggested for TrkB-T1. ${ }^{26}$

We were interested in the identification of additional neuronal survival pathways downregulated in stroke and sought to characterize their contribution to excitotoxicity. Therefore, we analyzed TrkB expression in models of ischemia and excitotoxicity, and characterized mechanisms acting at the mRNA and protein levels yielding TrkB-FL downregulation and TrkB-T1 upregulation. We investigated the contribution of this imbalance to excitotoxicity and ischemia, and demonstrated a strong association with neurodegeneration. Analysis of human necropsies suggested that TrkB levels might be similarly altered by stroke. These results identified the TrkB isoforms as new therapeutic targets for neuroprotection against excitotoxicity.

\section{Results}

Opposite regulation of TrkB isoforms in a model of ischemia. To identify the neuronal survival pathways downregulated in ischemia, we analyzed the TrkB expression and neurodegeneration in a rat model of focal cerebral ischemia, induced by a 1-h middle cerebral artery occlusion (MCAO), followed by reperfusion for $6 \mathrm{~h}$. These conditions produced a moderate decrease in Nissl staining in this artery territory (Supplementary Figure S1) and allowed TrkB characterization in neurons committed to die, whereas avoiding massive death starting after $24 \mathrm{~h}$ of reperfusion. ${ }^{12,13,27}$ Immunohistochemistry with isoformspecific antibodies revealed high TrkB-T1 levels in NeuNlabeled neurons in the ischemic region compared with very low expression in equivalent regions of contralateral neocortex (Figure 1A). Analysis of glial fibrillary acidic protein (Supplementary Figures S2A and B) demonstrated

Figure 1 Transient cerebral ischemia induces the opposite regulation of TrkB-FL and T1 isoforms in rat neurons, and TrkB-T1 upregulation is associated with neurodegeneration. Double immunohistochemistry with isoform-specific antibodies of coronal sections of animals subjected to MCAO followed by blood reperfusion ( $6 \mathrm{~h}$ ), a model in which damage is limited to the cortex, revealed that neurons in the ischemic region present a large increase in TrkB-T1 levels compared with those in the contralateral hemisphere $(\mathbf{A})$ and an important decrease of TrkBFL expression (B). Combined immunostaining of TrkB-T1 and NeuN with FluoroJade $B(F J B)$ labeling demonstrated a dramatic association of TrkB-T1 upregulation in the ischemic region with early neuronal degeneration (arrows) (C). However, there was a small number of neurons in this area which did not express TrkB-T1 or stain with FJB (arrowheads). Representative confocal microscopy images corresponding to single sections are shown. The scale bars represent $10 \mu \mathrm{m}$
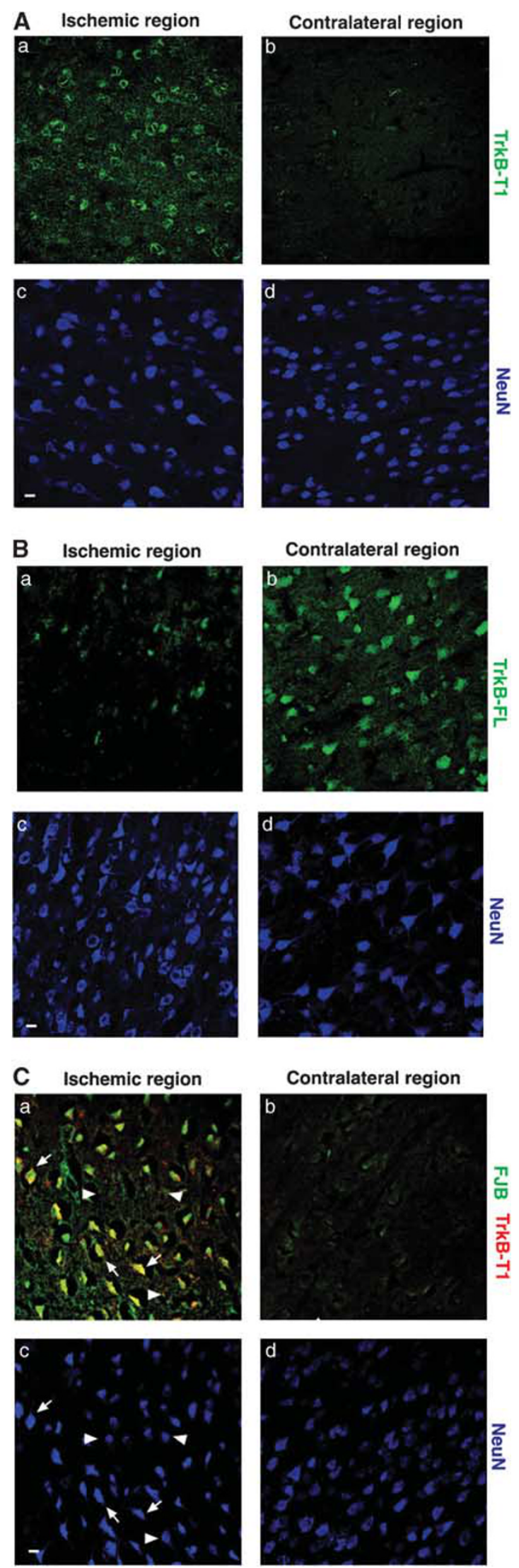
scarce astrocytic staining in the ischemic area at this early time and no TrkB-T1 upregulation in glial cells. It was important to establish the cells where TrkB-T1 increased, because this isoform is expressed in neurons and glial
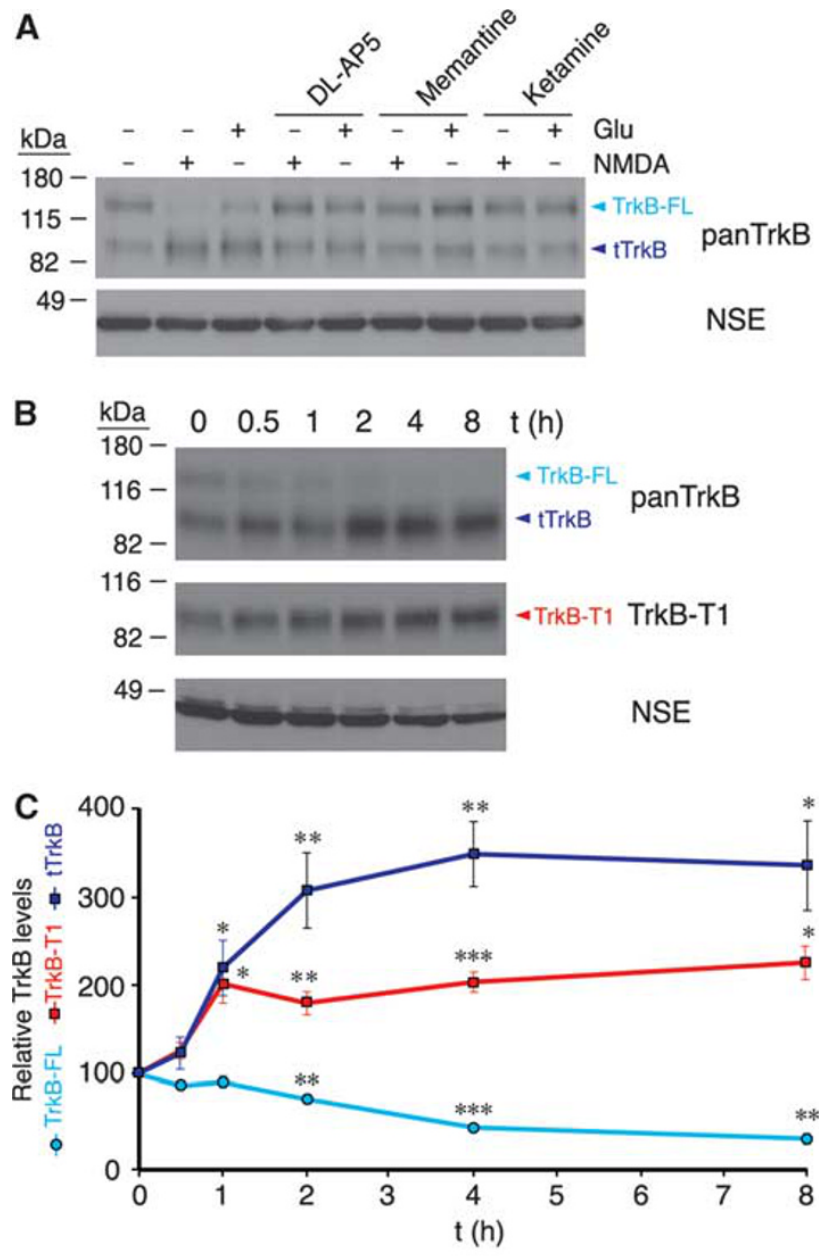

D Untreated
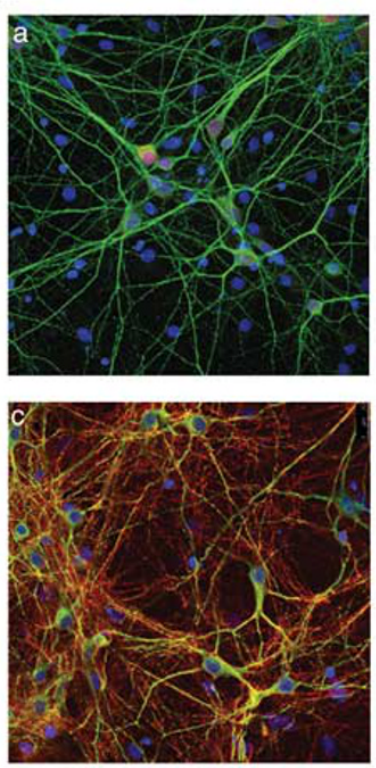

+ NMDA
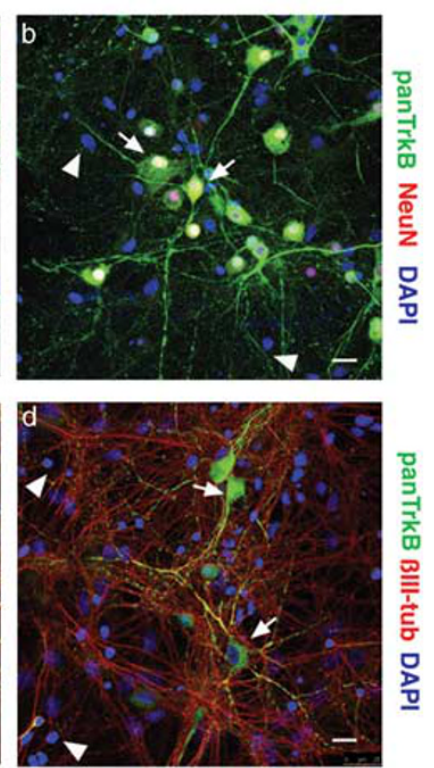

cells. ${ }^{28}$ Immunostaining with a TrkB-FL antibody demonstrated a dramatic reduction of the receptor in the ischemic area compared with the high levels found in neurons in the contralateral region (Figure 1B). The presence of neurons in the ischemic region was demonstrated with NeuN-labeling, which confirmed that TrkB-FL decrease was not due to neuronal death. The switch in TrkB expression from mostly TrkB-FL in contralateral regions to TrkB-T1 in ischemic tissue justified the labeling of both areas with an antibody directed to an epitope present in all TrkB isoforms (panTrkB; Supplementary Figures S2C and D). Finally, to investigate a possible association between TrkB-T1 expression and neurodegeneration, we combined immunostaining for TrkB-T1 and NeuN with Fluoro-Jade B-labeling, a compound that specifically binds to degenerating neurons. In the ischemic area, a dramatic association of high levels of TrkB-T1 expression with neurons undergoing degeneration was observed (Figure 1C, arrows).

Neuronal-specific TrkB regulation is induced by excitotoxicity. Excitotoxicity is a primary pathological mechanism of neurodegeneration in stroke. ${ }^{1}$ We investigated if excitotoxicity might be causing the TrkB-FL/TrkB-T1 imbalance by using a model reproducing in vitro the pathological activation of glutamate receptors. Primary cultures of rat cortices grown 14 days in vitro (DIV) were incubated with high concentrations $(100 \mu \mathrm{M})$ of NMDA or glutamate for $2 \mathrm{~h}$, conditions of relatively high-neuronal viability (Supplementary Figure S3). The NMDAR agonists induced a significant decrease in TrkB-FL (145 kDa) and a reciprocal increase in the truncated forms (collectively designated as tTrkB), as shown by panTrkB (Figure 2A). Other neuronal proteins such as the neuronal-specific enolase (NSE) were not modified by treatment, which demonstrated that TrkB regulation is specific and not a consequence of massive cell death. These effects required the specific activation of NMDARs and were blocked by competitive (DL-AP5) or non-competitive (memantine,

\footnotetext{
Figure 2 Neuronal-specific regulation of TrkB is induced by NMDAR overactivation in a cellular model of excitotoxicity. (A) Opposite regulation of the TrkB isoforms was induced by agonists of glutamate receptors and prevented by specific NMDAR antagonists. Primary cortical cultures (14 DIV) were treated with NMDA $(100 \mu \mathrm{M})$ or glutamate $(100 \mu \mathrm{M})$ with their co-agonist glycine $(10 \mu \mathrm{M})$ for $2 \mathrm{~h}$, with or without the antagonists DL-AP5 $(200 \mu \mathrm{M})$, memantine $(10 \mu \mathrm{M})$, or ketamine $(500 \mu \mathrm{M})$. TrkB-FL and the other truncated isoforms (tTrkB) that are recognized by panTrkB are indicated. (B) Time course of the TrkB-FL decrease and the TTrkB or TrkB-T1 increase in cultures treated with NMDA for the indicated times. (C) Quantitation of TrkB dysregulation. Relative protein levels of TrkB-FL, tTrkB, or TrkB-T1 (mean \pm S.E.M., $n=4$ experiments) were established after normalization to NSE and comparison with the protein levels in untreated cells, which were arbitrarily assigned a value of $100 \%$. The effect of NMDA was assessed using a Student's unpaired $t$-test $\left({ }^{\star} P<0.05,{ }^{* \star} P<0.01\right.$, and $\left.{ }^{* \star *} P<0.001\right)$. (D) Double immunofluorescence of cultures stimulated with NMDA for $1 \mathrm{~h}$ using panTrkB or neuronal-specific antibodies demonstrated the neuronal specificity of TrkB expression (arrows) and the lack of TrkB staining in glial cells in the culture (arrowheads). Analysis with isoform-specific antibodies was not possible, because they produced very weak immunofluorescent signals. Representative confocal microscopy images corresponding to single sections are shown. The scale bars represent $10 \mu \mathrm{m}$
} 
ketamine) NMDAR-antagonists (Figure 2A). The GluN2Bselective antagonist ifenprodil prevented TrkB regulation similar to DL-AP5 (Supplementary Figures S4A and B), consistent with the coupling of GluN2B subunits of the extrasynaptic NMDARs to excitotoxic pathways. ${ }^{2,29}$ Also supporting the importance of the source of calcium, the increase of intracellular calcium concentration with thapsigargin, an inhibitor of the endoplasmic reticulum $\mathrm{Ca}^{2+}$-ATPase, or calcium ionophore A23187 could not mimic the NMDA-induced TrkB regulation (Supplementary Figure S4C). Our mixed cultures, grown in minimun essential medium to reproduce the cellular brain environment and containing a $27 \pm 4 \%$ of astrocytes, required NMDAR overactivation with NMDA concentrations $>10 \mu \mathrm{M}$ for TrkB regulation (Supplementary Figures S5A, left panel, and S5B). In comparison, neuronal-enriched cultures in neurobasal medium containing a $4 \pm 0.4 \%$ of astrocytes presented a higher sensitivity for TrkB-FL downregulation (Supplementary Figure S5B) and neuronal death (Supplementary Figure $\mathrm{S} 5 \mathrm{C}$ ), probably reflecting reduced glutamate uptake.

A kinetic analysis of TrkB regulation demonstrated that $2 \mathrm{~h}$ of NMDA treatment significantly reduced the TrkB-FL expression to $72 \pm 5 \% \quad(P=0.004$; Figures $2 \mathrm{~B}$ and $\mathrm{C})$. Stimulation over 4 and $8 \mathrm{~h}$ further decreased TrkB-FL to $45 \pm 2 \%(P=0.0001)$ and $33 \pm 3 \%(P=0.001)$, respectively. Conversely, we observed a progressive increase in tTrkB levels (Figure 2B, upper panel), which reached values of $219 \pm 30 \%(P=0.01)$ and $304 \pm 39 \%(P=0.006)$, relative to untreated cultures 1 and $2 \mathrm{~h}$ after NMDA stimulation, respectively (Figure $2 \mathrm{C}$ ). This increase was partially due to the upregulation of TrkB-T1 $(95 \mathrm{kDa}$; Figure 2B, middle panel), which reached values of $196 \pm 18 \%(P=0.01) 1 \mathrm{~h}$ after NMDA stimulation, relative to untreated cultures, and increased more gradually afterwards (Figure 2C). We analyzed the cell specificity of TrkB regulation by immunofluorescence of neuronal markers NeuN and $\beta$ III-tubulin, and panTrkB (Figure 2D). Total TrkB was mainly detected in the soma and dendrites of untreated neurons, also labeled with NeuN (Figure 2D, panel a) or $\beta$ III-tubulin (Figure 2D, panel c). After NMDA stimulation for $1 \mathrm{~h}$, a condition that induced prevalence of tTrkB over TrkB-FL (Figure 2B), neurons were still labeled by panTrkB (Figure 2D, arrows), a result resembling that obtained in the ischemic brain (Supplementary Figures S2C and D). Morphological changes characteristic of excitotoxicity and typically preceding death, ${ }^{30}$ such as dendritic focal swelling and the formation of varicosities, were found in stimulated neurons (Figure 2D, panels $b$ and $d$ ).

\footnotetext{
Mechanisms of TrkB regulation induced by excitotoxicity. Transient ischemia modifies brain patterns of alternative mRNA splicing. ${ }^{31}$ Therefore, we examined changes in the relative mRNA levels of TrkB isoforms after excitotoxic stimulation indicative of a modification of mRNA splicing. Quantitative RT-PCR revealed that TrkB-FL transcripts were significantly reduced to $59 \pm 5 \%$ $(P=0.00001)$ and $43 \pm 12 \% \quad(P=0.04)$, compared with untreated cells after 2 and $3 \mathrm{~h}$ of NMDA stimulation, respectively (Figure 3a). Conversely, TrkB-T1 transcripts were increased significantly after treatment and reached
}

values of $146 \pm 13 \%(P=0.005)$ by $2 \mathrm{~h}$ of stimulation. We concluded that excitotoxicity induced a progressive increase in the ratio of TrkB-T1 to TrkB-FL mRNA.

We considered other possible mechanisms of TrkB regulation, such as endocytic degradation of BDNF/TrkB complexes that might be mediated by BDNF released after excitotoxic stimulation. ${ }^{32}$ Compared with NMDA, BDNF treatment (1-4 h) did not extensively modify TrkB levels, though it induced TrkB tyrosine-kinase phosphorylation and activation (Figure $3 \mathrm{~b}$ ). Quantitation confirmed a significant regulation of TrkB isoforms induced by NMDA compared with stable levels after BDNF treatment, with only a small and transient increase of TrkB-T1 at $1 \mathrm{~h}$ (Figure $3 \mathrm{c}$ ). These results suggest that, in the time window analyzed, BDNF-induced mechanisms did not contribute to TrkB regulation by NMDA. As TrkB-interacting proteins (e.g. NMDARs and kinase D-interacting substrate of $220 \mathrm{kDa}$ /ankyrin repeat-rich membrane spanning (Kidins220/ ARMS) $)^{33}$ are calpain substrates, ${ }^{13,14,27}$ and this protease is activated by excitotoxicity, ${ }^{16}$ we investigated the possible contribution of calpain to TrkB-FL downregulation. The NMDA-induced decrease in TrkB-FL was largely prevented by the calpain inhibitor calpeptin, which had no effect on TrkBT1 upregulation (Figure $3 \mathrm{~d}$ ). We tested calpain activity and calpeptin efficacy by analyzing the processing of a wellcharacterized calpain substrate, FL brain spectrin $(240 \mathrm{kDa})$, which produces breakdown products of 150 and $145 \mathrm{kDa}$. In contrast, lactacystin did not prevent TrkB-FL regulation, although it increased p53 levels (Figure 3d), a proteasome substrate. The contribution of calpain to Trk-FL downregulation was quantified. Preincubation with calpeptin upon NMDA treatment greatly restored TrkB-FL levels, which were $68 \pm 5 \%$ of those obtained in cells incubated only with calpeptin, and significantly higher than those in NMDA-treated neurons (39 $\pm 3 \% ; \quad P=0.0003$, calpeptin + NMDA versus NMDA; Figure 3e). A parallel comparison failed to show an effect of calpain inhibition in TrkB-T1 regulation $(212 \pm 26 \%$ versus $222 \pm 56 \%$, respectively). Collectively, these experiments revealed a dual mechanism of TrkB regulation in excitotoxicity; one major component was extensive calpaindependent processing of TrkB-FL, and the other element was due to regulation of the relative TrkB-FL and TrkB-T1 mRNA levels.

Characterization of TrkB-FL calpain processing. To further characterize the TrkB-FL processing, we generated a dual lentiviral vector that expresses an $\mathrm{N}$-terminally hemagglutinin (HA)-tagged TrkB-FL and GFP (LV-HA-TrkBFL/GFP) or two fluorescent proteins (LV-DsRed/GFP) ${ }^{34}$ in the same neuron (Figure 4A). These genes were under the control of synapsin promoters, which drove their efficient neuro-specific co-expression. ${ }^{34}$ Immunostaining of cultures infected with a low multiplicity of LV-HA-TrkB-FL/GFP confirmed expression of the transducible genes. Compared with non-transduced cells, an increase in TrkB was found only in neurons that expressed GFP (Figure 4B). Immunoblot analysis of cultures infected with increasing multiplicities of LV-HA-TrkB-FL/GFP demonstrated augmented total TrkB$F L$ levels compared with constant levels of endogenous TrkB-FL in LV-DsRed/GFP-infected cells (Figure 4C). Recombinant TrkB-FL receptors were processed after 
a

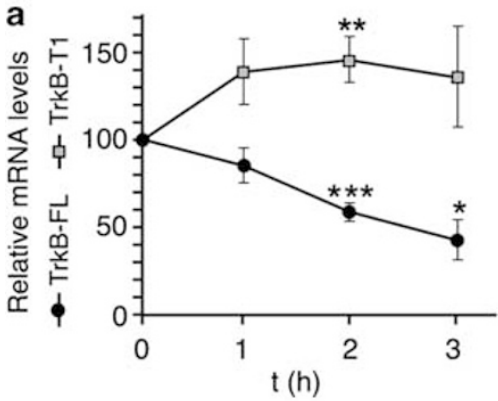

b

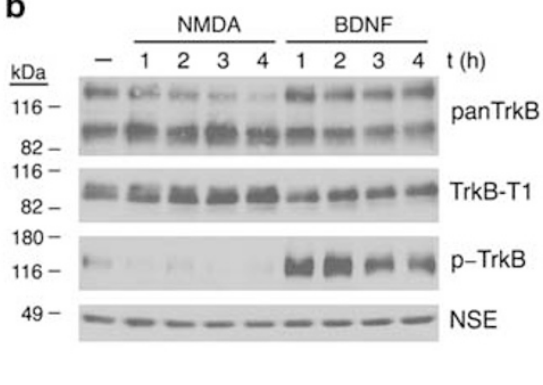

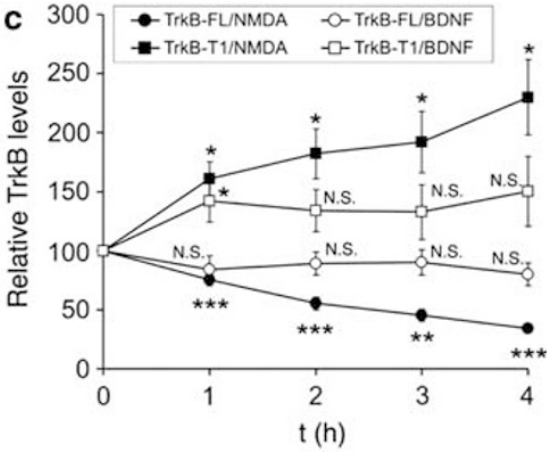

d

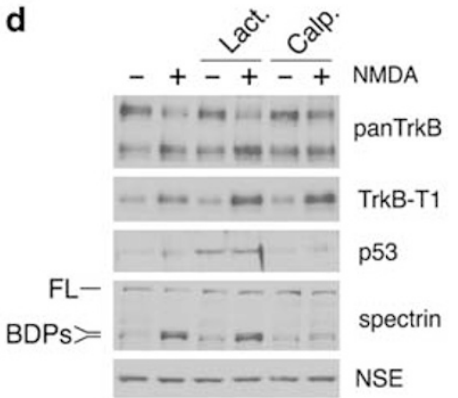

e

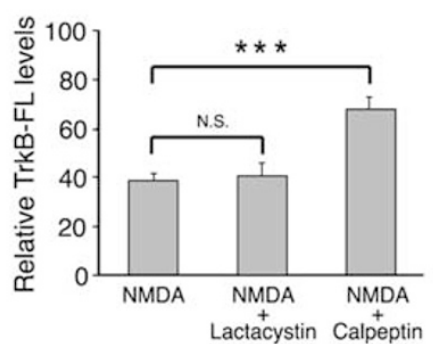

Figure 3 Two different mechanisms contribute to TrkB regulation in excitotoxicity. (a) Quantitative RT-PCR of TrkB-FL and TrkB-T1 mRNAs. Relative mRNA levels (mean \pm S.E.M., $n=3$ experiments) were established after normalization to $18 \mathrm{~S} r R N A$ and comparison with untreated cells, which were arbitrarily assigned a value of $100 \%$. The effect of NMDA was assessed using a Student's unpaired $t$-test $\left({ }^{\star} P<0.05,{ }^{* \star} P<0.01\right.$, and $\left.{ }^{\star \star \star} P<0.001\right)$. Reverse regulation of isoform mRNAs producing a progressive increase in the fraction of TrkB-T1 mRNA was observed. (b) Contrary to NMDA stimulation, treatment with BDNF $(100 \mathrm{ng} / \mathrm{ml})$ for $1-4 \mathrm{~h}$ induced TrkB activation, established by phospho (p)-TrkB detection with p-TrkA (Tyr490)-specific antibodies, but no general modification of TrkB-FL, tTrkB, or TrkB-T1. (c) Quantitation of TrkB-FL and TrkB-T1 levels in cultures treated with NMDA and BDNF showed that BDNF-induced mechanisms were not contributing to TrkB regulation induced by excitotoxicity. Levels of TrkB-FL and TrkB-T1 (mean \pm S.E.M., $n=10$ experiments) were compared with the levels in untreated neurons, which were arbitrarily assigned a value of $100 \%$. Student's unpaired $t$-test was used to evaluate the differences between treated or untreated cultures (N.S., nonsignificant; ${ }^{*} P<0.05,{ }^{* \star} P<0.01$, and ${ }^{* \star *} P<0.001$ ). (d) TrkB-FL is processed by a calpain-dependent mechanism during excitotoxicity. Cultures were preincubated with protease inhibitors lactacystin $(20 \mu \mathrm{M})$ or calpeptin $(10 \mu \mathrm{M})$ before NMDA treatment for $2 \mathrm{~h}$. The processing of FL brain spectrin to produce characteristic breakdown products (BDPs) was used as a standard assay of calpain activity, whereas levels of p53 were analyzed to confirm proteasome inhibition. (e) Quantitation of TrkB-FL revealed calpain-dependent processing as the major mechanism of NMDA-induced TrkB regulation. Cultures were preincubated and treated with lactacystin or calpeptin as described above. Normalized TrkB-FL levels (mean \pm S.E.M., $n=8$ experiments) in neurons treated with NMDA, with or without protease inhibitors, were compared with the corresponding levels found in control neurons without NMDA, which were arbitrarily assigned a value of $100 \%$. Student's unpaired $t$-test was used to evaluate the differences in the NMDA-treated cultures between those with or without calpain inhibitor preincubation (N.S., nonsignificant; ${ }^{* \star \star} P<0.001$ )

NMDA treatment and yielded fragment(s) that contained the $\mathrm{N}$-terminal HA-tag with a mobility of $\sim 95 \mathrm{kDa}$ (Figure 4D). Calpeptin prevented HA-TrkB-FL cleavage, demonstrating that the processing of recombinant and endogenous receptors was calpain-dependent.

We also analyzed in vitro processing of TrkB by purified calpain (Figure 4E). Incubation of extracts containing HATrkB-FL with calpain revealed that, contrary to TrkB-T1, recombinant and endogenous TrkB-FL were sensitive to cleavage. This result directly established TrkB-FL as a novel calpain substrate and showed that cleavage occurred downstream but nearby the divergence point with TrkB-T1 sequence. This TrkB-FL fragment, together with TrkB-T1, contributed to the increase of tTrkB detected by panTrkB in NMDA-treated neurons (Figure 2B). In conclusion, calpain processing generated a truncated TrkB-FL that lacked the tyrosine-kinase domain and was strikingly similar to TrkB-T1.

Upregulation of TrkB-T1 in human stroke. To approach the possibility that TrkB neuronal survival pathways are also downregulated in human ischemia, we analyzed necropsies from acute ischemic stroke patients, where we compared infarcted cerebral cortex to equivalent regions in the contralateral hemispheres. We characterized TrkB-T1 by immunoblot and found very high levels in the ischemic area compared with contralateral tissue, whereas similar levels of other neuronal proteins (e.g. NSE) were found in both the hemispheres (Figure 5A, left panel). Quantitation of these results showed a mean increase of normalized TrkB-T1 levels of 4.8 times in the infarcted tissue versus the contralateral hemisphere (Figure $5 \mathrm{~A}$, right panel), which was not significant, probably because of inter-individual variability, differences in time of tissue collection $(2.5-7 \mathrm{~h}$ postmortem), and small size of the sample analyzed $(n=3)$. For TrkB-FL, we found heterogeneous levels in the control tissues (Figure 5A, left panel) probably due to different levels of spontaneous postmortem calpain activation taking place early after death in infarcted and, more importantly, not infarcted tissue. ${ }^{35}$ Accordingly, almost complete cleavage of spectrin was observed in control and ischemic tissues. To reproduce the instability of TrkB-FL in the postmortem tissue, we analyzed the isoform levels in the brain of the rat 
A

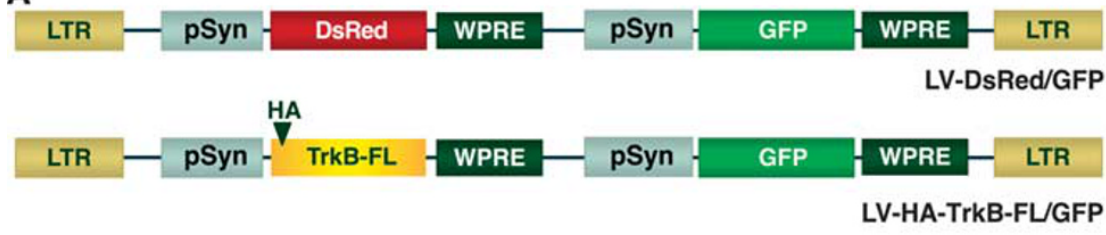

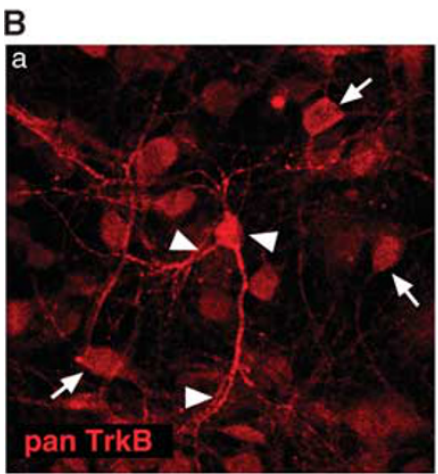

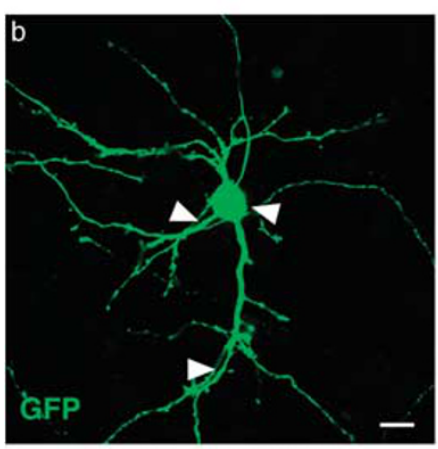

LV-HA-TrkB-FL/GFP

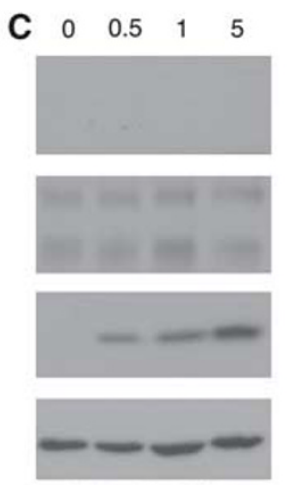

LV-DsRed/GFP $\begin{array}{lllll}0 & 0.5 & 1 & 5 & \text { m.o.i. }\end{array}$

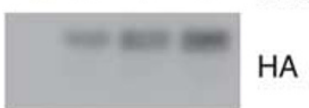

$\mathrm{HA}$

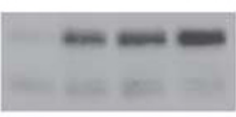

panTrkB

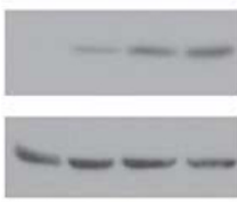

LV-HA-TrkB-FLGFP

D

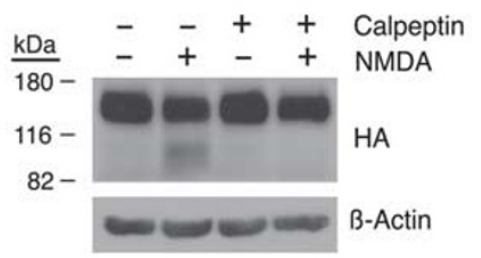

\section{E}

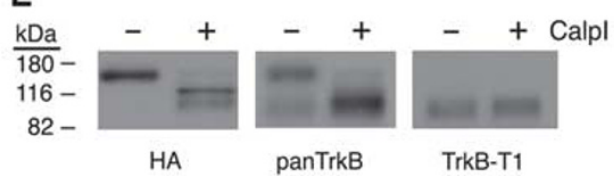

Figure 4 Characterization of TrkB-FL as a calpain substrate. (A) Schematic diagram of lentiviral vectors LV-DsRed/GFP (encoding DsRed and GFP fluorescent proteins) and LV-HA-TrkB-FL/GFP (coding for HA-TrkB-FL and GFP). The recombinant proteins are regulated by neuro-specific synapsin promoters (pSyn), and their sequences are followed by woodchuck hepatitis post-transcriptional regulatory element (WPRE) for the enhancement of expression. LTR, long terminal repeat. (B) Double immunostaining with panTrkB and GFP antibodies of cultures infected with a low multiplicity of LV-HA-TrkB-FL/GFP demonstrated increased TrkB levels in specific neurons co-expressing GFP (arrowheads) compared with endogenous TrkB in uninfected neurons (arrows). Confocal microscopy images correspond to single sections. The scale bars represent $10 \mu \mathrm{m}$. (C) Cultures infected with lentivirus LV-HA-TrkB-FL/GFP using increasing multiplicities of infection (m.o.i.) showed the expression of HA-TrkB-FL and the specific increase of TrkB-FL total levels compared with LV-DsRed/GFP-infected cells. (D) Processing of recombinant HA-TrkB-FL expressed in LV-HA-TrkB-FL/GFP-infected neurons $(m .0 . i=2)$ is induced by $2 \mathrm{~h}$ of NMDA treatment and produces N-terminally HA-tagged fragments. Cleavage is blocked by pre-incubation for $1 \mathrm{~h}$ with the specific calpaininhibitor calpeptin $(10 \mu \mathrm{M})$. (E) In-vitro calpain processing of extracts prepared from neurons infected with LV-HA-TrkB-FL/GFP using purified calpain I (80 U/ml) directly established TrkB-FL as a calpain substrate, in contrast to TrkB-T1, and suggested that cleavage yields a truncated TrkB-FL that is similar to TrkB-T1. The additional HA-TrkB-FL fragment that was produced in vitro, with a mobility $\sim 110 \mathrm{kDa}$ (left panel), may correspond to an intermediate product and was not observed in cultures subjected to excitotoxicity

embryos kept at room temperature for different periods after death (2-8h; Figure 5B). Processing of TrkB-FL was fast and largely parallel to that of spectrin, although the levels of TrkB-T1 were more stable. This isoform could be still detected by $8 \mathrm{~h}$ postmortem, a time point when TrkB-FL and spectrin were already processed. These results suggest that, in stroke, TrkB-FL downregulation induced by ischemia can be only observed at the earliest postmortem times (Figure 5A, patient 15), and that TrkB-T1 upregulation is not related to spontaneous calpain activation. Next, we performed immunohistochemistry of human infarcted cortex and confirmed the increase in TrkB-T1 compared with the very low levels found in the contralateral hemisphere
(Figure 5C). The identification of post-mitotic neurons with NeuN antibodies revealed that TrkB-T1 upregulation in stroke was mainly neuro-specific (Figure $5 \mathrm{C}$ ) as demonstrated before in the animal model (Figure $1 \mathrm{~A}$ and Supplementary Figures S2A and B). The heterogeneity of NeuN levels in the ischemic region was suggestive of neurodegeneration, which may be associated with TrkB-T1 upregulation.

Recovery of TrkB-FL/TrkB-T1 balance confers neuroprotection against excitotoxicity. Dysregulation of TrkB isoforms induced by excitotoxicity may contribute to neuronal death and, therefore, reversion of these changes 


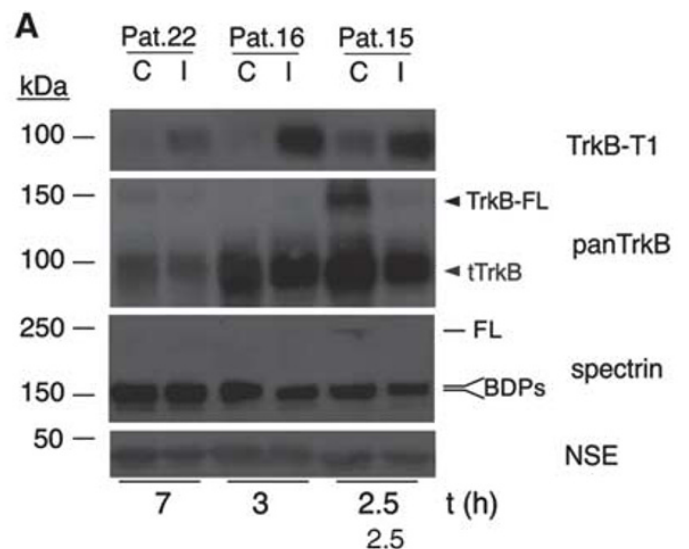

C
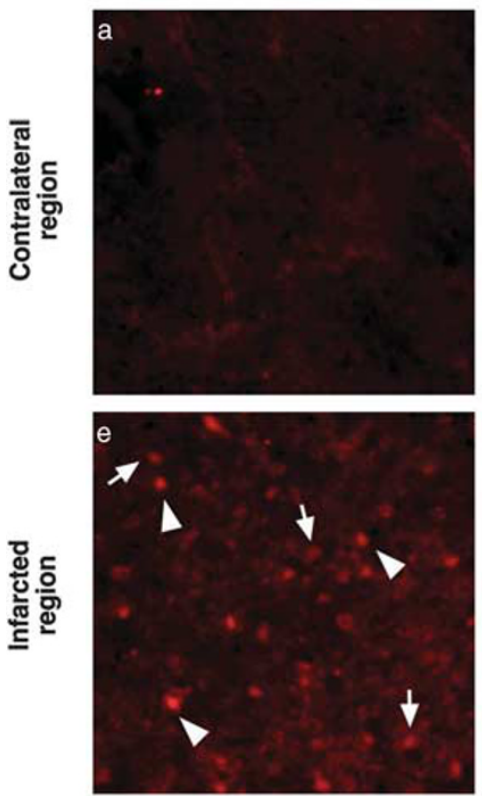

NeuN
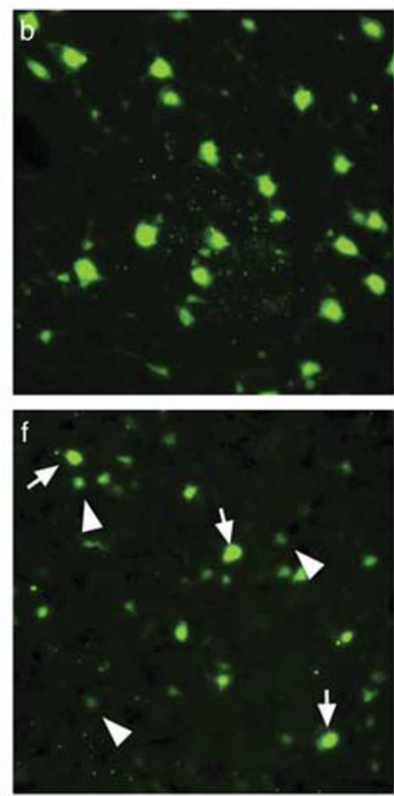

B

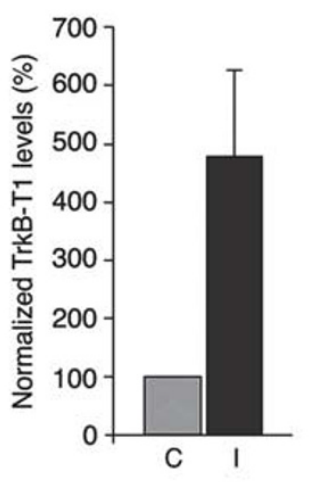

Figure 5 TrkB-T1 isoform levels increase after stroke in human brain neurons. (A) Immunoblot analysis of TrkB-T1 expression in the cerebral cortex of three patients using an isoform-specific antibody. A comparison of the infarcted cortex (I) with the corresponding area of the contralateral hemisphere (C) demonstrated a dramatic increase of TrkB-T1 in the ischemic area, but similar levels of NSE in both regions. The postmortem time of tissue collection for each patient is indicated below the blot panels. Heterogeneous TrkB-FL levels in the control tissue of the various patients were observed using a panTrkB antibody that recognized the FL isoform and the truncated forms of the TrkB receptor (tTrkB). The processing of FL brain spectrin to produce breakdown products (BDPs) demonstrated the activation of this protease in necropsies corresponding to both control and ischemic tissue. The histogram represents the quantitation of the normalized TrkB-T1 levels (mean \pm S.E.M., $n=3$ ) in the infarcted tissue compared with the contralateral hemisphere, arbitrarily assigned a value of $100 \%$. Student's unpaired $t$-test established that the observed differences were nonsignificant $(P=0.1)$. (B) Demonstration of TrkB-FL cleavage in parallel to spontaneous calpain activation in normal brain tissue at different times after death. Individual Wistar rat embryos (E18) were kept at ambient temperature for 2 to $8 \mathrm{~h}$ before dissection of brain cortices, which were then compared with tissue collected immediately after death. No increase in TrkB-T1 levels was observed, showing that calpain activation and TrkB-T1 upregulation are not related processes. (C) Immunohistochemistry of tissue sections corresponding to infarcted cerebral cortex or equivalent regions in the contralateral hemisphere confirmed the increased expression of TrkB-T1 in the ischemic area, mostly in post-mitotic neurons labeled with a NeuN antibody. Heterogeneity of NeuN levels in the infarcted area is highlighted. Although neurons that have low TrkB-T1 levels are strongly stained by NeuN (arrows), high TrkB-T1 levels are associated with neurons that have low NeuN expression (arrowheads). The scale bar represents $50 \mu \mathrm{m}$

would confer neuroprotection. To test this hypothesis, we investigated the consequences in neuronal viability of an increase in TrkB-FL expression. After LV-HA-TrkB-FL/GFP infection, the combined levels of wild-type and recombinant TrkB-FL were higher compared with the LV-DsRed/GFPinfected cultures (Figure 6a), although both the TrkB-FL forms were still processed by NMDA-activated calpain. This increase only partially counteracted one of the mechanisms of TrkB regulation induced by excitotoxicity and had no effect on TrkB-T1 upregulation (Figure 6a) or neuronal viability (Figure 6b). Next, we explored the effect of counteracting the TrkB-T1 increase by using microRNAs (miRNAs). Five double-stranded oligonucleotides (Supplementary Figure S6) encoding pre-miRNAs specific for target sequences in TrkB-T1 mRNA 3'-UTR (Supplementary Figure S7A) were used to produce $\mathrm{pcD} / \mathrm{miT} 1$ plasmids, which were initially tested in a heterologous system (Supplementary Figure S7B). Plasmids showing the highest efficiency in TrkB-T1 interference 


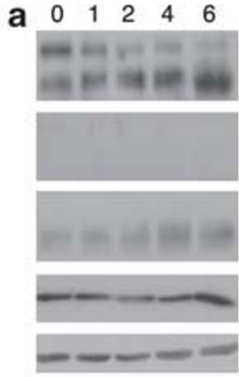

LV-DsRed/GFP $\begin{array}{llllll}0 & 1 & 2 & 4 & 6 & \mathrm{t}(\mathrm{h})\end{array}$

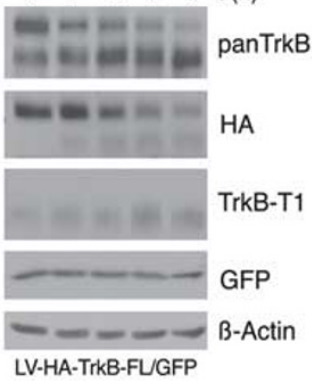

b

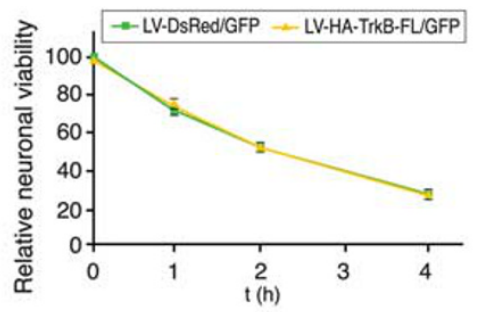

C

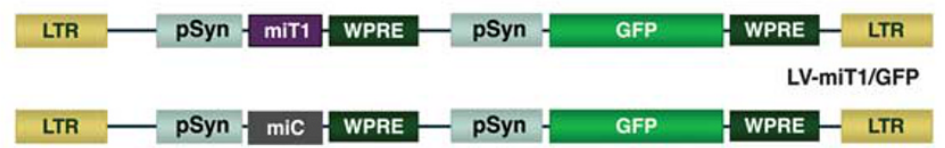

LV-mic/GFP

d

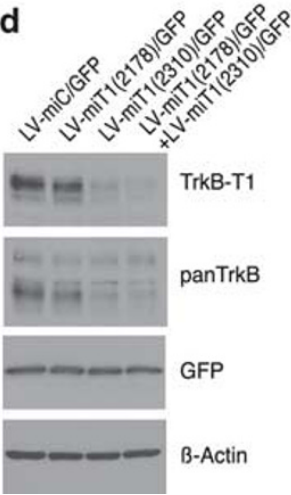

e

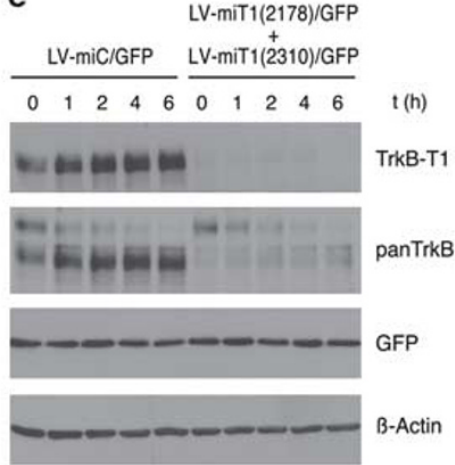

f

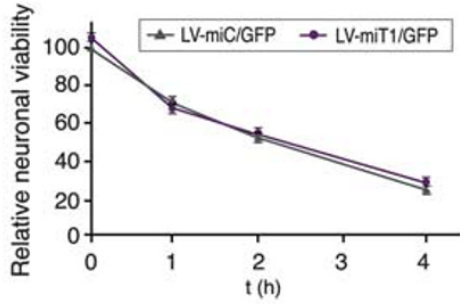

Figure 6 Effect of increased TrkB-FL expression or TrkB-T1 interference during excitotoxicity. (a) Time course of NMDA treatment in cultures transduced with LV-HATrkB-FL/GFP (m.o.i. = 2) demonstrated higher levels of total TrkB-FL compared with LV-DsRed/GFP-infected cells. (b) Relative neuronal viability (mean \pm S.E.M., $n=6$ ) of cells infected and NMDA treated as above. Using Student's unpaired t-test, no significant differences were found between cells infected with LV-HA-TrkB-FL/GFP or LV-DsRed/GFP. (c) Schematic diagram of lentiviral vectors for neuro-specific interference of the TrkB-T1 isoform. Pre-miRNAs specific for target sequences in TrkB-T1 mRNA, starting at nucleotide 2178 or 2310, were cloned under the control of pSyn to produce viruses LV-miT1(2178)/GFP and LV-miT1(2310)/GFP (herein, LV-miT1/GFP). Transcription of miRNAs was controlled by RNA polymerase II and was cell type-specific. Lentivirus LV-miC/GFP contains negative-control pre-miRNAs sequences. (d) Efficient and specific interference of basal TrkB-T1 expression was obtained by double infection with LV-miT1(2178)/GFP plus LV-miT1(2310)/GFP (m.0.i. = 1 for each virus) or LV-miT1(2310)/GFP with LV-miC/GFP (m.o.i. =1 for each virus). No changes were observed for TrkB-FL or other analyzed proteins. (e) Double infection with LV-miT1(2178)/GFP and LV-miT1(2310)/GFP strongly interfered with the TrkB-T1 upregulation that was induced by NMDA compared with cultures infected with LV-miC/GFP (m.o.i. =2). (f) Relative neuronal viability (mean \pm S.E.M., $n=6$ ) of cells infected with LV-miT1(2178)/GFP and LV-miT1(2310)/GFP (m.o.i. $=1$ for each virus), or LV-mic/GFP $(m .0 . i .=2)$, treated or not treated with NMDA. Student's unpaired $t$-test revealed no significant differences in viability between cells infected with these viruses

compared with the $\mathrm{pcD} / \mathrm{miC}$ control $(\mathrm{pcD} / \mathrm{miT} 1(2310)$ and $\mathrm{pcD} / \mathrm{miT1}(2178)$ ) were chosen for further analysis. To produce neuro-specific TrkB-T1 interference, these pre-miRNAs were cloned under the control of a synapsin promoter to produce lentiviruses LV-miT1(2178)/GFP and LV-miT1(2310)/GFP (Figure 6c). Strong interference of basal TrkB-T1 expression was produced by LV-miT1(2310)/GFP-infection, which was further increased by mixed infection with LV-miT1(2178)/GFP, a virus having only moderate effects by itself (Figure 6d). Interference was specific for TrkB-T1 and not observed for TrkB-FL or other proteins. Additionally, the combined infection with LV-miT1/ GFP viruses strongly prevented the TrkB-T1 upregulation induced by excitotoxicity in LV-miC/GFP-infected cells (Figure 6e). However, it had no effect on TrkB-FL downregulation (Figure 6e) or neuronal viability (Figure 6f).

We reasoned that it might be necessary to simultaneously counteract both the mechanisms of TrkB dysregulation to protect neurons from excitotoxic damage. Therefore, we generated another lentivirus that produced TrkB-FL and the two more efficient TrkB-T1 pre-miRNAs (LV-HA-TrkB-FL/ $2 x m i T 1$ ) in the same neuron (Figure 7a). This virus strongly interfered with basal TrkB-T1 expression and increased TrkBFL levels compared with untreated LV-DsRed/GFP-infected cells (Figure 7b). Moreover, after NMDA stimulation, LV-HATrkB-FL/2xmiT1 prevented TrkB-T1 upregulation and induced higher TrkB-FL levels compared with LV-DsRed/ GFP-infected cultures (Figure 7b). We compared the effect of double counteraction of TrkB dysregulation on neuronal viability with respect to single strategies. The viability of untreated cultures infected with lentiviruses LV-DsRed/GFP, LV-HA-TrkB-FL/2xmiT1, LV-HA-TrkB-FL/GFP, LV-miC/GFP, or LV-miT1/GFP was very similar (Supplementary Figure S8), suggesting that an increase in TrkB-FL and/or a decrease in TrkB-T1 did not enhance basal survival pathways. In contrast, infected neurons responded differently to $2 \mathrm{~h}$ of NMDA 
a

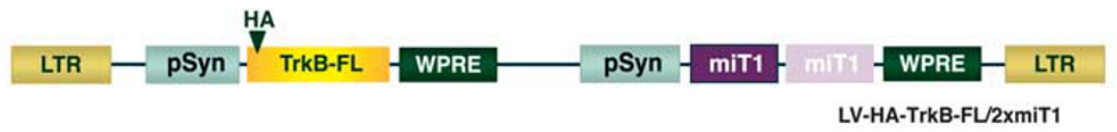

b

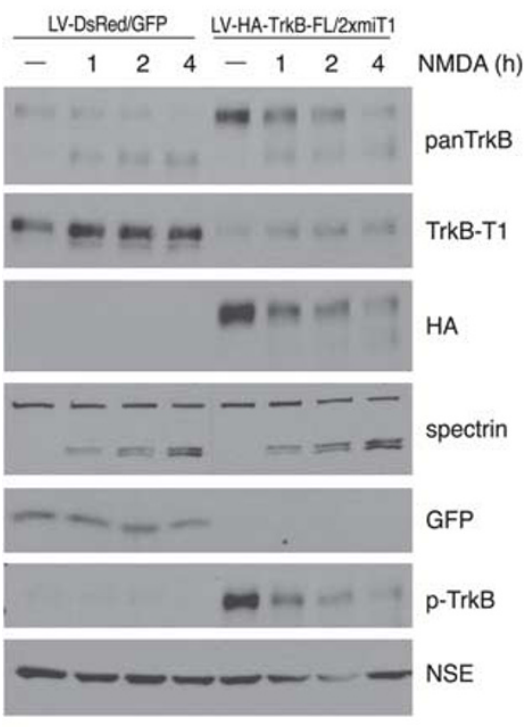

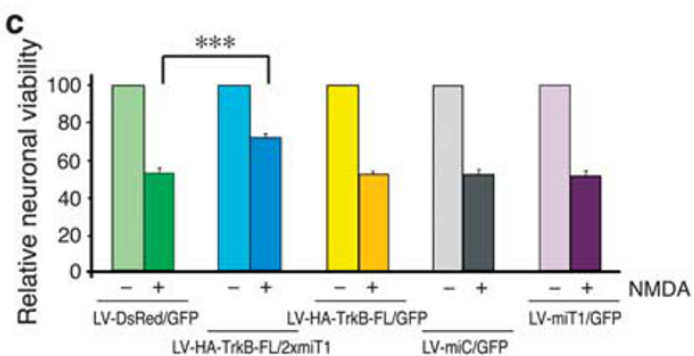

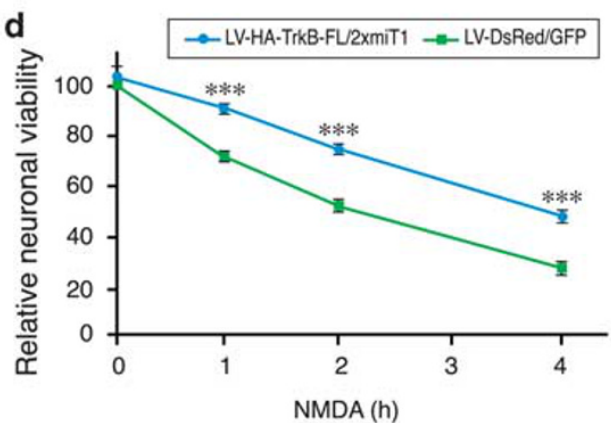

Figure 7 Increased TrkB-FL expression together with TrkB-T1 interference confers neuroprotection against excitotoxicity. (a) Schematic diagram of lentivirus LV-HA-TrkB$\mathrm{FL} / 2 \mathrm{xmiT1}$, which was designed for the neuro-specific expression of recombinant HA-TrkB-FL simultaneously with the double interference of TrkB-T1 by miT1(2178) and miT1(2310) pre-miRNAs (purple and violet boxes, respectively). (b) Time course of NMDA treatment in cultures transduced with LV-HA-TrkB-FL/2xmiT1 (m.0.i. $=2$ ) showing higher levels of total TrkB-FL and strong interference of TrkB-T1 both in basal and stimulated conditions compared with LV-DsRed/GFP infections. (c) Relative neuronal viability (mean \pm S.E.M., $n=3$ ) of cultures transduced with different viruses that modified the TrkB-FL/TrkB-T1 balance and stimulated with NMDA ( $2 \mathrm{~h}$ ) are expressed as percentages of the values obtained in untreated cells infected with the same virus (arbitrarily established as 100\%). Cultures were infected with LV-HA-TrkB-FL/2xmiT1, LV-DsRed/GFP, LV-HA-TrkB-FL/GFP, LV-miC/GFP (m.o.i. =2), or LV-miT1(2178)/GFP, together with LV-miT1(2310)/GFP (m.o.i. =1 for each virus). Student's unpaired $t$-test demonstrated statistically significant differences between cells infected with LV-HA-TrkB-FL/2xmiT1 or LV-DsRed/GFP, and treated with NMDA (** $P<0.001)$. (d) Timecourse analysis of the effect of NMDA on neuronal viability after LV-HA-TrkB-FL/2xmiT1 infection (m.o.i. $=2$ ). Relative neuronal viability (mean \pm S.E.M., $n=3$ ) is expressed as percentage of the value obtained in untreated LV-DsRed/GFP infected cells (arbitrarily established as 100\%). Student's unpaired $t$-test showed significant differences between cells infected with LV-HA-TrkB-FL/2xmiT1 or LV-DsRed/GFP at different times of NMDA treatment ( $\left.{ }^{\star \star \star} P<0.001\right)$, which demonstrates a neuroprotective effect of the simultaneous increase in TrkB-FL expression and the interference with TrkB-T1 synthesis

treatment. Neuronal viability was reduced by NMDA to $75 \pm 1 \%$ in LV-HA-TrkB-FL/2xmiT1-infected cells, a value that was significantly higher than that obtained in cultures infected with LV-DsRed/GFP $(52 \pm 3 \%, P=0.0001)$ or the other viruses (Figure 7c). A time-course analysis in LV-HATrkB-FL/2xmiT1-infected neurons demonstrated that the greatest differences in the kinetics of neuronal death were obtained during the initial period of excitotoxicity, though neuronal viability was higher at the various times tested, compared with LV-DsRed/GFP-infected cells (Figure 7d). After $4 \mathrm{~h}$ of NMDA stimulation, the viability of LV-HA-TrkB-FL/ $2 x m i T 1$-infected cultures was $48 \pm 3 \%$, a value significantly higher than that found in the LV-DsRed/GFP-infected cultures $(27 \pm 2 \%, P=0.0005)$. As protection was observed in LV-HATrkB-FL/2xmiT1-infected neurons, we analyzed TrkB activity finding high levels of phosphorylated TrkB-FL in untreated cultures that decreased progressively over the time of NMDA treatment similar to total TrkB-FL (Figure 7b). Therefore, these results demonstrated that the dysregulation of normal TrkB-FL/TrkB-T1 balance is an important mechanism of neuronal death induced by excitotoxicity, and the simultaneous increase in TrkB-FL expression and interference of TrkB-T1 synthesis has a neuroprotective effect against these insults.

\section{Discussion}

Excessive activation of NMDARs is central to degeneration and death of the adult neurons in ischemia and other disorders ${ }^{1}$ by mechanisms that are not completely understood. Here, we unveiled a TrkB-FL/TrkB-T1 imbalance as one neurodegeneration mechanism in ischemia. A dysregulation of TrkB was observed in neurons of infarcted human brain and rat neurons that were subjected to in vivo or in vitro excitotoxicity. One of the mechanisms contributing to the TrkB regulation altered the physiological ratio of mRNAs encoding for TrkB isoforms and favored the expression of TrkB-T1 over TrkB-FL. Splicing and transcription are tightly coupled processes, and splice-site selection is altered by changes in chromatin structure, ${ }^{36}$ transcription rates, ${ }^{37}$ and promoter identity and occupancy. ${ }^{38}$ As TrkB is a CREB-regulated gene, ${ }^{9,10}$ the suppression of CREB by extrasynaptic NMDAR 
stimulation $^{2}$ may decrease $\operatorname{TrkB}$ transcription and shift the splicing of TrkB precursor mRNA. However, we will need to consider other possible mechanisms induced by excitotoxicity. The second and major mechanism of TrkB regulation was calpain processing of TrkB-FL, unveiled here as a new calpain substrate. A detailed analysis of TrkB-FL truncation in stroke patients was hampered by postmortem spontaneouscalpain activation in non-infarcted tissue. ${ }^{35}$ However, in a cellular model, we were able to establish that calpain activation by excitotoxicity generated a truncated TrkB-FL receptor similar to TrkB-T1, which may act as an additional dominantnegative protein. All together, the decrease of BDNF/TrkB signaling will impact neuronal survival and brain function.

Ischemia also regulates other proteins critical to neuronal survival related to TrkB. The scaffolding protein PSD-95, which is central to NMDAR regulation, is cleaved by calpain, together with GluN2A and GluN2B. This combined processing results in a truncated NMDAR defective in pro-survival signaling. ${ }^{13}$ Another example is Kidins220/ARMS, ${ }^{33,39}$ a protein that interacts with NMDARs ${ }^{27}$ or ephrin and neurotrophin receptors ${ }^{39}$ and has a key role in regulating neuronal survival in excitotoxicity and ischemia. ${ }^{27}$ The downregulation of TrkB in addition to that of NMDARs and Kidins220/ARMS implies some redundancy in the mechanisms of excitotoxic death, both concerning the number of proteins involved in a pathway and the existence of overlapping mechanisms for some proteins. This redundancy suggests functional relevance for the efficient silencing of specific pro-survival signaling cascades during the excitotoxic response.

These results identify TrkB isoforms as potential therapeutic targets for neuroprotection in ischemia and should be considered for the rational design of therapies for stroke and related disorders. An enhancement of BDNF/TrkB signaling has been proposed as a possible treatment for stroke; ${ }^{19}$ however, this approach might only prevent the delayed death of neurons still expressing TrkB-FL after stroke. Here, we explored the recovery of the TrkB-FL/TrkB-T1 balance as a novel therapeutic strategy in stroke. Using lentiviruses capable of neuro-specific expression of recombinant proteins and/or miRNAs, we demonstrated the need for a combined strategy to increase TrkB-FL expression and TrkB-T1 interference to produce a significant and reproducible protection from excitotoxic insults. The recovery of neuronal viability in cultures infected with LV-HA-TrkB-FL/2xmiT1 was limited by the calpain processing of recombinant HA-TrkB-FL and the redundancy of excitotoxic mechanisms (i.e. other death pathways remained active). Because of the complexity of the excitotoxic process, we will need to design combined strategies that counteract the regulation of different targets that are important for neuronal survival.

\section{Materials and Methods}

Plasmids. Plasmid pRc-CMV-HA-TrkB-FL, provided by Y Barde (Biozentrum, Basel, Switzerland), encodes rat TrkB-FL with a HA epitope inserted at the N-terminus. Oligonucleotides $5^{\prime}$-GGGGACGCCTGGATCCGCGTAGGGACACGC ACTCTG-3' and 5'-GACAAGGAGCGGCCGCCCGGGGTGAGGCAGTGC-3' were used to introduce BamHI and Nott sites flanking this CDNA. Nucleotides mutated in the TrkB-FL CDNA are in bold, and restriction sequences are underlined. This BamHI-Notl cDNA fragment was sub-cloned, substituting DsRed in LV-DsRed/ GFP $^{34}$ digested with the same enzymes to produce LV-HA-TrkB-FL/GFP. In this dual lentiviral vector, synthesis of HA-TrkB-FL and GFP is under the control of neuro-specific synapsin promoters and their sequences are followed by woodchuck hepatitis post-transcriptional regulatory elements for expression enhancement.

miRNAs for the specific knockdown of rat TrkB-T1 expression were designed using the RNAi-designer website from Invitrogen (Carlsbad, CA, USA). We selected five different double-stranded oligonucleotides (see details in Supplementary Figure S6) coding pre-miRNAs specific for target sequences in the $3^{\prime}$-UTR of the mRNA (Supplementary Figure S7A). These sequences were cloned into the pcDNA6.2-GW/miRNA vector using a Block-iT Polll miR RNAi expression vector kit (Invitrogen) to generate the $\mathrm{pcD} / \mathrm{miT1}$ plasmids. This kit also provided a $\mathrm{pcDNA6.2-}$ $\mathrm{GW} / \mathrm{miR}$-negative control plasmid (herein $\mathrm{pcD} / \mathrm{miC}$ ). After preliminary evaluation of interference efficiency in a heterologous system (Supplementary Figure S7B), vectors suitable for neuro-specific TrkB-T1 interference were prepared by taking advantage of the fact that miRNA transcription is controlled by RNA polymerase II and is cell type-specific. Thus, the BamHI-Xhol fragments isolated from the $\mathrm{pcD} /$ miT1 plasmids that produced efficient TrkB-T1 interference $(\mathrm{pcD} / \mathrm{miT1}(2178)$ and $\mathrm{pcD} / \mathrm{miT1}(2310))$ and $\mathrm{pcD} / \mathrm{miC}$ were cloned into the lentiviral vector LV-GFP (syn-syn-GFP) ${ }^{34}$ and digested with the same enzymes to produce LV-miT1(2178)/ GFP, LV-miT1(2310)/GFP, and LV-miC/GFP. We also generated plasmid pcD/ 2xmiT1 for double TrkB-T1 interference by cloning the BamHI-Xhol fragment that was isolated from $\mathrm{pcD} / \mathrm{miT1}(2310)$ into $\mathrm{pcD} / \mathrm{miT1}(2178)$ digested with $\mathrm{Bgll}$ and $X$ Xol. Finally, we designed a vector suitable for double TrkB-T1 interference and HA-TrkB-FL expression in the same neurons. We eliminated the GFP gene from LV-HA-TrkB/GFP by first digesting with EcoRI and, because two Nhel sites are present in this plasmid, partial digestion with Nhel afterwards. Annealed oligonucleotides 5'-CTAGCGTGGATGATCAAGGTGTGTCTTGTTAGTGGGTCTT ATGTCTCGAGGTGGTG-3' and 5'-AATTCACCACCTCGAGACATAAGACCCACT AACAAGACACACCTTGATCATCCACG- $3^{\prime}$ were cloned into the EcoRI and Nhel sites to produce LV-HA-TrkB/oligo, in which we introduced unique $B C l$ and $X$ hol sites (underlined in the sequences). Then, we cloned the BamHI-Xhol fragment from $\mathrm{pcD} / 2 \times \mathrm{xmiT1}$, which carried the two TrkB-T1 miRNAs, into LV-HA-TrkB/oligo that was digested with Bcll and Xhol to generate LV-HA-TrkB-FL/2xmiT1.

Primary neuronal culture. Primary cultures were prepared from the cerebral cortex of 18-day-old Wistar rat embryos, and grown in minimun essential medium (Invitrogen-Life Technologies, Carslbad, CA, USA) as described previously. ${ }^{12}$ As they are mixed cultures, we inhibited glial growth by adding $\operatorname{AraC}(10 \mu \mathrm{M})$ after 7 DIV. When indicated, primary cultures were also grown in neurobasal medium (Invitrogen-Life Technologies) with serum-free B-27 supplement. Cultures were treated with different reactives at 14 DIV. Unless otherwise stated, NMDA (100 $\mu \mathrm{M})$ and glycine $(10 \mu \mathrm{M})$ were used for NMDAR overactivation. For protease inhibition, cultures were pretreated with the indicated compound for $30 \mathrm{~min}$ before NMDA addition, and the inhibitor remained in the medium for the duration of the NMDA treatment. Infection of primary cultures with lentiviruses produced as described in Supplementary Materials and Methods was performed at 7 DIV and proceeded until 14 DIV. ${ }^{13}$

Experimental model of cerebral ischemia. All animal procedures were performed in compliance with the European Community Law 86/609/EEC and were approved by the Consejo Superior de Investigaciones Científicas Committee. Male Sprague-Dawley adult rats $(275-300 \mathrm{~g})$ were anesthetized, maintained, and monitored during surgery for MCAO as described previously. ${ }^{12}$ The MCA was ligated before its bifurcation into frontal and parietal branches. After complete interruption of blood flow, both common carotid arteries were also occluded, and all three arteries were maintained in this state for $60 \mathrm{~min}$. Six hours after blood reperfusion, animals were intracardially perfused with cold $4 \%$ paraformaldehyde (wt/vol) in PBS, pH 7.4. Brains were post-fixed for $6 \mathrm{~h}$ at $4{ }^{\circ} \mathrm{C}$ and cryoprotected by serial immersion in 10,15 , and $20 \%$ sucrose (wt/vol) in PBS for $6 \mathrm{~h}$ at $4{ }^{\circ} \mathrm{C}$. Frozen coronal sections ( $30 \mu \mathrm{m}$ thick) were prepared using a cryostat (Leica, Wetzlar, Germany). In this model, the characteristic changes of ischemic damage are limited to the cortex, and the subcortical structures and basal ganglia are spared.

Human brain samples. This study was approved by the Vall d'Hebron Hospital Ethics Committee (PR[HG]85/04), and informed consent was obtained from relatives before autopsy. The study included three deceased patients (two men) who had an ischemic stroke within the previous 4 days (range 24-96 h). On autopsy and during macroscopic examination, an experienced neuropathologist, guided with recent neuroradiology images, established the infarcted area in the cerebral cortex, mainly by consistency and parenchyma color, and surgically separated the gray matter. Brain tissue from equivalent regions in the ipsilateral 
(infarction) and contralateral hemispheres was collected within the first $7 \mathrm{~h}$ after death, snap-frozen in liquid nitrogen, and stored at $-80^{\circ} \mathrm{C}$ for immunoblotting or fixed with formalin for immunohistochemistry.

Immunoblot analysis. For human brain extracts, tissue was homogenized in lysis buffer $(50 \mathrm{mM}$ Tris-HCl (pH 7.6), $150 \mathrm{mM} \mathrm{NaCl}, 5 \mathrm{mM} \mathrm{CaCl}, 0.05 \%$ Brij-35 ( $\mathrm{vol} / \mathrm{vol}), 0.02 \% \mathrm{NaN}_{3}$ ( $\left.\mathrm{vol} / \mathrm{vol}\right)$, and $1 \%$ Triton X-100 (vol/vol)) containing protease inhibitors (1 $\mathrm{mM}$ phenylmethylsulfonyl fluoride and $7 \mu \mathrm{g} / \mathrm{ml}$ aprotinin). Cortical cultures were lysed in RIPA buffer as described previously, ${ }^{12}$ and equal amounts of protein $(25-50 \mu \mathrm{g})$ were analyzed by immunoblotting, using the primary and secondary antibodies indicated in the Supplementary Information. Immunocomplexes were detected with a Bioluminescence kit from Perkin-Elmer (Boston, MA, USA). Densitometric analysis was performed with $\mathrm{NIH}$ Image analysis software. The results were normalized to levels of NSE in the same samples and are represented as relative values in reference to the values obtained in the untreated cells.

Immunofluorescence. The immunofluorescence procedure was described previously. ${ }^{13}$ Confocal images were acquired using a Radiance 2000 confocal (BioRad, Hercules, CA, USA) coupled to an inverted Axiovert S100 TV microscope (Zeiss, Oberkochen, Germany) with a 63x Plan-Apochromat oil immersion objective.

Immunohistochemistry. Frozen sections $(12 \mu \mathrm{m})$ of human specimens were prepared using a cryostat, post-fixed in ice-cold acetone for $15 \mathrm{~min}$, and washed in $0.05 \%$ Tween (vol $/ \mathrm{vol}$ )-PBS. Sections were blocked with $10 \% \mathrm{BSA}$ (wt/vol), $0.3 \%$ (vol/vol) Triton X-100 (vol/vol) in PBS for $1 \mathrm{~h}$ at room temperature, and incubated overnight at $4{ }^{\circ} \mathrm{C}$ with primary antibodies. After incubation with secondary antibodies, sections were mounted on coverslips using Vectashield with DAPI (Vector Laboratories, Burlingame, CA, USA) and analyzed with a fluorescence microscope (Olympus IX71, Essex, UK) interfaced with an image analysis system. Images were processed with ImageJ software (NIH). In the MCAO model, Nissl $(0.1 \%$ (wt/vol) cresyl violet) staining permitted the identification of neocortex ischemic tissue. Adjacent sections $(30 \mu \mathrm{m})$ were processed for immunohistochemistry as described previously. ${ }^{13}$ Sections were mounted in Fluoromount-G solution (Southern Biotechnology Assoc., Birmingham, AL, USA) or stained with Fluoro-Jade B (Chemicon, Temacula, CA, USA) according to manufacturer's instructions. Confocal images were acquired as described for immunofluorescence.

Calpain in vitro proteolysis. Protein extracts were prepared using RIPA buffer containing $1 \mathrm{mM}$ phenylmethylsulfonyl fluoride, $10 \mu \mathrm{g} / \mathrm{ml}$ pepstatin, $10 \mu \mathrm{g} / \mathrm{ml}$ aprotinin, $1 \mathrm{mM}$ EDTA, and $1 \mathrm{mM}$ EGTA. Calpain I $(80 \mathrm{U} / \mathrm{ml})$ was added to diluted extracts $(50 \mu \mathrm{g})$ supplemented with $5 \mathrm{mM}$ DTT and $2.5 \mathrm{mM} \mathrm{CaCl}_{2}$, and incubated $30 \mathrm{~min}$ at $37^{\circ} \mathrm{C}$

Statistical analysis. Graph data represent mean values and the S.E.M obtained from three or more independent experiments. Statistical significance was performed using an unpaired $t$-test (Student $t$-test). $P$-values $<0.05$ were considered statistically significant.

\section{Conflict of Interest}

The authors declare no conflict of interest.

Acknowledgements. We thank Y Barde (Biozentrum, Basel, Switzerland) and M Barbacid (CNIO, Madrid, Spain) for donating plasmids and JM Cosgaya (CSIC, Madrid, Spain) for providing purified BDNF. This work was supported by Grants BFU2007-67695 and BFU2010-18380 from Ministerio de Ciencia e Innovación (MICINN), PIE 200420E601 and 2007201012 from CSIC to MD-G, and BFU2004-01498 to AR-P. The Neurovascular Research Laboratory participates in the Spanish Stroke Research Network RENEVAS (RD06/0026/0010) and receives funding from FIS (08/361). OGV and EC were recipients of fellowship-contracts 'Formación de Personal Investigador' (FPI) conferred by MICINN and FIS (05/322), respectively. We are grateful to T Iglesias, C López-Menéndez and B Jiménez for critical reading of this manuscript and helpful discussions.

1. Choi DW. Glutamate neurotoxicity and diseases of the nervous system. Neuron 1988; 1 : 623-634.
2. Hardingham GE, Fukunaga $Y$, Bading $H$. Extrasynaptic NMDARs oppose synaptic NMDARs by triggering CREB shut-off and cell death pathways. Nat Neurosci 2002; 5: 405-414.

3. Papadia S, Stevenson P, Hardingham NR, Bading H, Hardingham GE. Nuclear Ca2+ and the CAMP response element-binding protein family mediate a late phase of activity-dependent neuroprotection. J Neurosci 2005; 25: 4279-4287.

4. Mori $H$, Mishina M. Structure and function of the NMDA receptor channel. Neuropharmacology 1995; 34: 1219-1237.

5. Papadia S, Soriano FX, Leveille F, Martel MA, Dakin KA, Hansen HH et al. Synaptic NMDA receptor activity boosts intrinsic antioxidant defenses. Nat Neurosci 2008; 11: 476-487.

6. Thomas GM, Huganir RL. MAPK cascade signalling and synaptic plasticity. Nat Rev Neurosci 2004; 5: 173-183.

7. Shieh PB, Hu SC, Bobb K, Timmusk T, Ghosh A. Identification of a signaling pathway involved in calcium regulation of BDNF expression. Neuron 1998; 20: 727-740.

8. Tao X, Finkbeiner S, Arnold DB, Shaywitz AJ, Greenberg ME. Ca2+ influx regulates BDNF transcription by a CREB family transcription factor-dependent mechanism. Neuron 1998; 20: 709-726.

9. Kingsbury TJ, Murray PD, Bambrick LL, Krueger BK. Ca(2+)-dependent regulation of TrkB expression in neurons. J Biol Chem 2003; 278: 40744-40748.

10. Deogracias R, Espliguero G, Iglesias T, Rodríguez-Peña A. Expression of the neurotrophin receptor trkB is regulated by the CAMP/CREB pathway in neurons. Mol Cell Neurosci2004; 26: $470-480$.

11. Stanika RI, Pivovarova NB, Brantner CA, Watts CA, Winters CA, Andrews SB. Coupling diverse routes of calcium entry to mitochondrial dysfunction and glutamate excitotoxicity. Proc Natl Acad Sci USA 2009; 106: 9854-9859.

12. Gascón S, Deogracias R, Sobrado M, Roda JM, Renart J, Rodríguez-Peña A et al. Transcription of the NR1 subunit of the N-methyl-D-aspartate receptor is down-regulated by excitotoxic stimulation and cerebral ischemia. J Biol Chem 2005; 280: 35018-35027.

13. Gascón S, Sobrado M, Roda JM, Rodríguez-Peña A, Díaz-Guerra M. Excitotoxicity and focal cerebral ischemia induce truncation of the NR2A and NR2B subunits of the NMDA receptor and cleavage of the scaffolding protein PSD-95. Mol Psychiatry 2008; 13: 99-114.

14. Simpkins KL, Guttmann RP, Dong Y, Chen Z, Sokol S, Neumar RW et al. Selective activation induced cleavage of the NR2B subunit by calpain. J Neurosci 2003; 23: 11322-11331.

15. Vosler PS, Brennan CS, Chen J. Calpain-mediated signaling mechanisms in neuronal injury and neurodegeneration. Mol Neurobiol 2008; 38: 78-100.

16. Bevers MB, Neumar RW. Mechanistic role of calpains in postischemic neurodegeneration. J Cereb Blood Flow Metab 2008; 28: 655-673.

17. Tezuka T, Umemori H, Akiyama T, Nakanishi S, Yamamoto T. PSD-95 promotes Fyn-mediated tyrosine phosphorylation of the N-methyl-D-aspartate receptor subunit NR2A. Proc Natl Acad Sci USA 1999; 96: 435-440.

18. Iwasaki $\mathrm{Y}$, Gay B, Wada K, Koizumi S. Association of the Src family tyrosine kinase Fyn with TrkB. J Neurochem 1998; 71: 106-111.

19. Moskowitz MA, Lo EH, ladecola C. The science of stroke: mechanisms in search of treatments. Neuron 2010; 67: 181-198.

20. Segal RA, Greenberg ME. Intracellular signaling pathways activated by neurotrophic factors. Annu Rev Neurosci 1996; 19: 463-489.

21. Middlemas DS, Lindberg RA, Hunter T. trkB, a neural receptor protein-tyrosine kinase: evidence for a full-length and two truncated receptors. Mol Cell Biol 1991; 11: 143-153.

22. Ninkina N, Adu J, Fischer A, Pinon LG, Buchman VL, Davies AM. Expression and function of TrkB variants in developing sensory neurons. Embo J 1996; 15: 6385-6393.

23. Carim-Todd L, Bath KG, Fulgenzi G, Yanpallewar S, Jing D, Barrick CA et al. Endogenous truncated TrkB.T1 receptor regulates neuronal complexity and TrkB kinase receptor function in vivo. J Neurosci 2009; 29: 678-685.

24. Dorsey SG, Bambrick LL, Balice-Gordon RJ, Krueger BK. Failure of brain-derived neurotrophic factor-dependent neuron survival in mouse trisomy 16. J Neurosci 2002; 22: 2571-2578.

25. Eide FF, Vining ER, Eide BL, Zang K, Wang XY, Reichardt LF. Naturally occurring truncated trkB receptors have dominant inhibitory effects on brain-derived neurotrophic factor signaling. J Neurosci 1996; 16: 3123-3129.

26. Michaelsen K, Zagrebelsky M, Berndt-Huch J, Polack M, Buschler A, Sendtner M et al. Neurotrophin receptors TrkB.T1 and p75NTR cooperate in modulating both functional and structural plasticity in mature hippocampal neurons. Eur $J$ Neurosci 2010; 32: 1854-1865.

27. López-Menéndez C, Gascón S, Sobrado M, Vidaurre OG, Higuero AM, Rodríguez-Pena A et al. Kidins220/ARMS downregulation by excitotoxic activation of NMDARs reveals its involvement in neuronal survival and death pathways. J Cell Sci 2009; 122: 3554-3565

28. Armanini MP, McMahon SB, Sutherland J, Shelton DL, Phillips HS. Truncated and catalytic isoforms of trkB are co-expressed in neurons of rat and mouse CNS. Eur J Neurosci 1995; 7: $1403-1409$

29. Tu W, Xu X, Peng L, Zhong X, Zhang W, Soundarapandian MM et al. DAPK1 interaction with NMDA receptor NR2B subunits mediates brain damage in stroke. Cell 2010; 140: 222-234.

30. Park JS, Bateman MC, Goldberg MP. Rapid alterations in dendrite morphology during sublethal hypoxia or glutamate receptor activation. Neurobiol Dis 1996; 3: 215-227. 
31. Daoud R, Mies G, Smialowska A, Olah L, Hossmann KA, Stamm S. Ischemia induces a translocation of the splicing factor tra2-beta 1 and changes alternative splicing patterns in the brain. J Neurosci 2002; 22: 5889-5899.

32. Jiang $\mathrm{X}$, Tian F, Mearow K, Okagaki $\mathrm{P}$, Lipsky RH, Marini AM. The excitoprotective effect of $\mathrm{N}$-methyl-D-aspartate receptors is mediated by a brain-derived neurotrophic factor autocrine loop in cultured hippocampal neurons. J Neurochem 2005; 94: 713-722.

33. Iglesias T, Cabrera-Poch N, Mitchell MP, Naven TJ, Rozengurt E, Schiavo G. Identification and cloning of Kidins220, a novel neuronal substrate of protein kinase D. J Biol Chem 2000; 275: 40048-40056.

34. Gascón S, Páez-Gómez JA, Díaz-Guerra M, Scheiffele P, Scholl FG. Dual-promoter lentiviral vectors for constitutive and regulated gene expression in neurons. J Neurosci Methods 2008; 168: 104-112.

35. Sorimachi Y, Harada K, Yoshida K. Involvement of calpain in postmortem proteolysis in the rat brain. Forensic Sci Int 1996; 81: 165-174.

36. Luco RF, Allo M, Schor IE, Kornblihtt AR, Misteli T. Epigenetics in alternative pre-mRNA splicing. Cell 2011; 144: 16-26.
37. Kornblihtt AR. Chromatin, transcript elongation and alternative splicing. Nat Struct Mol Biol 2006; 13: 5-7.

38. Xin $\mathrm{D}, \mathrm{Hu} \mathrm{L}$, Kong $\mathrm{X}$. Alternative promoters influence alternative splicing at the genomic level. PLOS ONE 2008; 3: e2377.

39. Kong H, Boulter J, Weber JL, Lai C, Chao MV. An evolutionarily conserved transmembrane protein that is a novel downstream target of neurotrophin and ephrin receptors. $J$ Neurosci 2001; 21: 176-185.

\section{(c)}

OMMERIEHIS RESER

Cell Death and Disease is an open-access journal published by Nature Publishing Group. This work is licensed under the Creative Commons Attribution-Noncommercial-No Derivative Works 3.0 Unported License. To view a copy of this license, visit http://creativecommons.org/licenses/by-nc-nd/3.0/

Supplementary Information accompanies the paper on Cell Death and Disease website (http://www.nature.com/cddis) 